\title{
Quantized structuring of transparent films with femtosecond laser interference
}

\begin{abstract}
Kitty Kumar ${ }^{1}$, Kenneth KC Lee ${ }^{2}$, Jianzhao Li $^{2}$, Jun Nogami ${ }^{1}$, Nazir P Kherani ${ }^{1,2}$ and Peter R Herman ${ }^{2}$
The confinement of laser interactions inside transparent materials assisted by tight optical focusing and short-pulsed nonlinear interactions has driven many high-resolution patterning and probing applications in science and technology. In thin transparent films, laser interactions confined to the film/substrate interface have underpinned blistering and ejection processes for nanofluidic channel fabrication, film patterning and cell catapulting. Here, we harness femtosecond lasers to drive nonlinear interactions within Fabry-Perot interference fringes to define narrow nanolength scale zones for highly resolved internal structuring of a film of refractive index, $n_{\text {film }}$, at fringe maxima separated by $\lambda / 2 n_{\text {film }}$. This novel interaction internally cleaves the film to open subwavelength internal cavities and form thin membranes at single or multiple depths from which follow significant opportunities for writing multilevel nanofluidic channels inside the film, as well as ejecting nanodisks at quantized film depths for coloring and three-dimensional surface patterning that promise new compact types of lab-in-film devices.
\end{abstract}

Light: Science \& Applications (2014) 3, e157; doi:10.1038/lsa.2014.38; published online 14 March 2014

Keywords: blistering; dielectrics; laser processing; quantized etching; subwavelength processing; thin films

\section{INTRODUCTION}

Laser material processing below the diffraction limit of light is a challenging and active area of research and development. Ultrashortpulsed lasers have dramatically improved the precision of light-matter interactions owing to greatly reduced thermal degradation, heat-affected zone, stochastic 'damage' threshold and strong nonlinear optical absorption that are widely studied and exploited today. ${ }^{1,2}$ Inside a transparent medium, femtosecond laser light can be confined in a volume smaller than the diffraction-limited radius and Rayleigh length of the focal volume created by high numerical aperture lenses. Multiphoton fluorescence can be locally excited to enable high resolution three-dimensional (3D) microscopy of living cells, ${ }^{3}$ while higher exposure in transparent dielectrics can induce gentle refractive index changes for writing into 3D optical circuits ${ }^{4}$ or driving microexplosions for 3D memory or marking. ${ }^{5,6}$

In a different approach, femtosecond laser light has been transmitted through a thin transparent film and confined to interact within the thin penetration depth of an underlying silicon substrate ${ }^{7,8}$ on length scales much smaller than the depth of focus. This narrow interaction zone explodes between the thin film and bulk substrate to form thin-film blisters and nanofluidic networks at the interface ${ }^{9}$ at low exposure, or the ejection of the whole film at high exposure. These ejection principles underlie the driving mechanisms in laserinduced forward transfer ${ }^{10}$ for patterning dielectrics, ${ }^{11}$ printing or additive manufacturing, ${ }^{12}$ releasing layers, ${ }^{13}$ matrix-assisted pulsed laser evaporation-direct write ${ }^{14}$ and cell ejection by laser pressure catapulting., ${ }^{3,15}$
Subdiffraction sized features on the half-wavelength scale of light, $\lambda$ l $2 n$, can also be recorded inside a transparent photosensitive material of refractive index, $n$, through the interference of light with itself, enabling holographic recordings in photographic film, Bragg gratings in optical fibers, ${ }^{16}$ and 3D photonic crystal templates in thick photoresist. ${ }^{17}$ In the case of thin transparent films, multisurface Fresnel reflections of laser light can also interfere and create a standing wave interference pattern with fringe maxima spaced by $\lambda / 2 n_{\text {film }}$ inside the film, where $n_{\text {film }}$ is the refractive index of the film. ${ }^{18}$ Such fringe effects were inferred by Hosokawa and co-workers ${ }^{19,20}$ to explain multistep etching of $\mathrm{Cu}$-phthalocyanine amorphous films driven by dissociation of weak intermolecular bonds. However, such fine patterning is not available for the majority of transparent materials such as dielectrics, requiring more aggressive laser interactions than photochemical response or intermolecular bond dissociation. To this end, Rudolf and co-workers associated a lower breakdown threshold for damage in single ${ }^{21,22}$ or multilayer ${ }^{23}$ dielectric films with the formation of such interference fringes, but with no evidence of spatially localized laser interaction directly observed inside the film. ${ }^{23}$ Hence, the concept of high resolution processing directly within transparent dielectric films is a largely unexplored area.

In this paper, we harness nonlinear absorption of femtosecond laser light to overcome thermal transport and enable a strongly localized laser-plasma formation within zones narrower than the optical fringes formed by thin film interference. These thin plasma disks produce for the first time material modification inside a dielectric film at a length scale much smaller than the focal Rayleigh range. The focal laser

${ }^{1}$ Department of Materials Science and Engineering, University of Toronto, Toronto, Canada and ${ }^{2}$ Department of Electrical and Computer Engineering, University of Toronto, Toronto, Canada

Correspondence: Dr PR Herman, Department of Electrical and Computer Engineering, University of Toronto, Toronto, Canada

E-mail: p.herman@utoronto.ca

Received 23 September 2013; revised 27 November 2013; accepted 1 December 2013 
interaction volume is divided into an array of thin axial planes that align with the Fabry-Perot fringe maxima predicted inside the dielectric film. The novel localized interaction can be controlled by laser exposure to modify the film interior periodically to either open internal nanovoids or to eject film disks in multiples of $\lambda / 2 n_{\text {film }}$ thickness. The temporal ejection of multiple disks by a single laser pulse was verified by time-resolved imaging of the ablation plume. These various interactions enable the fabrication of 3D nanofluidic structures inside the thin film, while the quantized surface structuring defines a new approach for film coloring and labeling or multilevel surface structuring. Together, the nanovoids and quantized ejection are attractive for structuring thin films that could significantly improve the functionality of complementary metal-oxide-semiconductor (CMOS), photovoltaics, Micro-electro-mechanical system (MEMS), Light-emitting diode (LED) and lab-on-a-chip devices where thin films are widely deployed during their manufacture, while also opening new directions for developing flexible electronic or display films or new lab-in-film concepts.

\section{MATERIALS AND METHODS}

SiN $_{x}$ films of 20-1545 nm thickness were grown by plasma-enhanced chemical vapor deposition (Oxford Instruments, Bristol, UK) on $\mathrm{Si}(001)$ wafers at $300{ }^{\circ} \mathrm{C}$ and $650 \mathrm{mT}$ chamber pressure using a gas mixture of $5 \%$ silane in nitrogen $(400 \mathrm{sccm})$, ammonia $(20 \mathrm{sccm})$ and nitrogen $(600 \mathrm{sccm})$. A deposition rate of $14 \mathrm{~nm} \mathrm{~min}^{-1}$ was obtained by cycling the radio frequency between $13.56 \mathrm{MHz}$ for $13 \mathrm{~s}$ and $100 \mathrm{kHz}$ for $7 \mathrm{~s}$ with respective power levels of $50 \mathrm{~W}$ and $40 \mathrm{~W}$.

A frequency doubled fiber laser (D-400-VR; IMRA, Ann Arbour, MI, USA) provided $200 \mathrm{fs}$ duration pulses at $522 \mathrm{~nm}$ wavelength and $100-\mathrm{kHz}$ repetition. A planoconvex lens $(f=8 \mathrm{~mm}$ focal length) focused the Gaussian-shaped beam $\left(M_{2}=1.33\right)$ to $\omega_{\mathrm{o}}=0.495 \mu \mathrm{m}$ radial $\left(1 / e^{2}\right)$ spot size onto the sample surface. Alternatively, the beam was masked and projected with an aspheric lens $(f=2.8 \mathrm{~mm})$ to a comparatively uniform $1.5 \mu \mathrm{m} \times 1.5 \mu \mathrm{m}$ square beam or $2.4 \mu \mathrm{m}$ diameter top-hat beam profile. Single or multiple laser pulses of 5 and $70 \mathrm{~nJ}$ each were selected with an acousto-optic modulator and delivered to isolated interaction sites or in patterns generated by scanning the sample with an XY motorized stage (ABL1000; Aerotech, Pittsburgh, PA, USA).

Time-resolved two-dimensional side-view images of the laser ablation plume were captured through a microscope objective $(50 \times)$ onto an intensified CCD camera (ICCD) (iStar DH734-18U-03), with time delays synchronized against the laser pulse. Plume emissions from $500 \mathrm{~nm}$ thick $\mathrm{SiN}_{x}$ films irradiated by 50-380 nJ pulse energy were recorded with 3-50 ns gate width and $0-2 \mu$ s time delay.

The morphology of the laser processed samples was inspected using scanning electron microscopy (SEM) and select samples were cut axially by a focused ion beam for cross-sectional SEM imaging.

\section{MODELING LASER INTERFERENCE INTERACTIONS}

Focused laser light entering a thin transparent film of thickness greater than $\lambda / 4 n_{\text {film }}$ will lead to formation of a Fabry-Perot interference pattern owing to Fresnel reflections and transmissions at the air/film and the film/substrate interface. Hence, the focal interaction volume will become divided into thin interaction zones as depicted in Figure 1a, which align with the fringe intensity maxima as shown in Figure $1 \mathrm{~b}$. The resulting fringe contrast can be controlled by tailoring the refractive index of the film and substrate materials. For the case of silicon nitride $\left(\mathrm{SiN}_{x} ; n_{\text {film }}=1.98\right)$ film of $d=500 \mathrm{~nm}$ thickness on a silicon substrate $\left(n_{\mathrm{si}}=4.192\right.$ and $\left.\kappa_{\mathrm{si}}=0.036\right)$, a $\lambda=522 \mathrm{~nm}$ wavelength laser beam incident from the top in Figure 1a will generate four fringe maxima $\left(m \approx d /\left(\lambda / 2 n_{\text {film }}\right)\right)$ on $\lambda / 2 n_{\text {film }}=131.8 \mathrm{~nm}$ period with a fringe visibility of 0.63 as shown in Figure $1 \mathrm{~b}$. A node is positioned near the $\mathrm{SiN}_{x} /$ silicon interface due to the out-of-phase Fresnel reflection, therefore locking the fringe pattern with the last fringe maximum positioned at $\lambda / 4 n_{\text {film }}=65.9 \mathrm{~nm}$ from the bottom interface. As a consequence, the position of the first fringe maximum from the top surface will vary with the film thickness, shown at $d-m \lambda$ l $2 n_{\text {film }}=38.7 \mathrm{~nm}$ distance from the air $/ \mathrm{SiN}_{x}$ interface for the case in Figure $1 \mathrm{~b}$.

At moderately low laser intensity, stronger linear interactions in the silicon substrate dominate over the nonlinear plasma excitation in the transparent film to drive laser heating only to a penetration depth of $1 /$ $\kappa_{\mathrm{si}}=28 \mathrm{~nm}$ in the silicon. The machining at the film/silicon interface

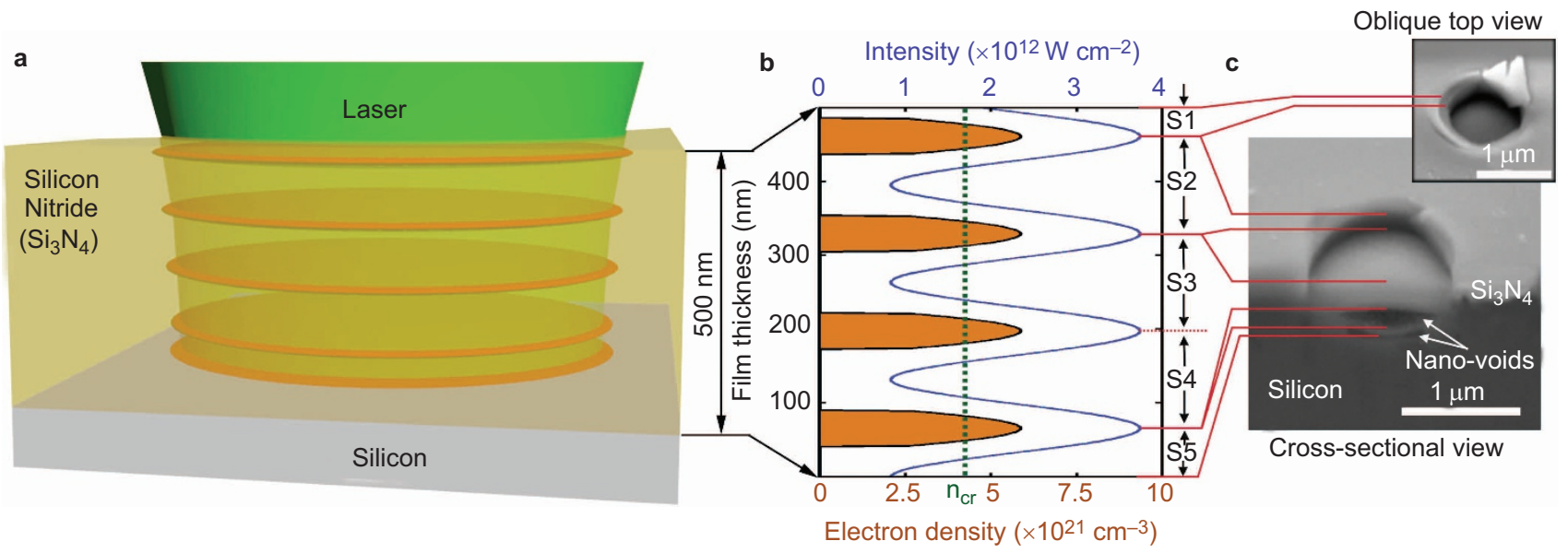

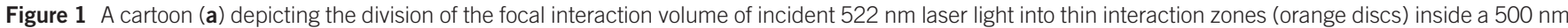

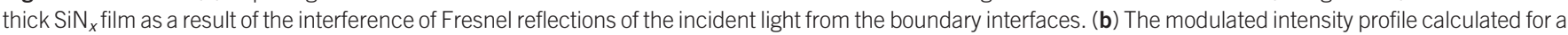

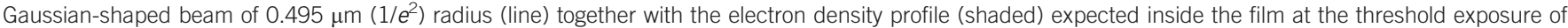

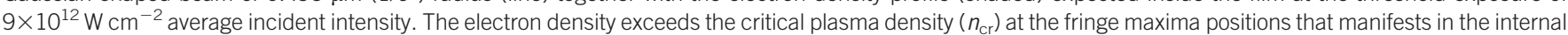

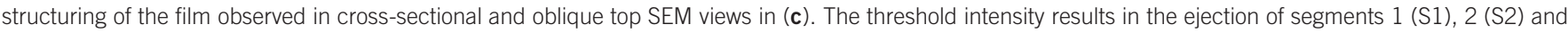

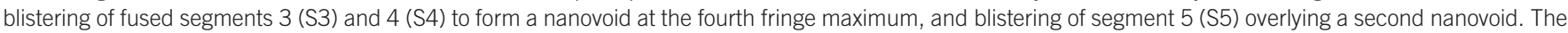
positions of the cleavage planes align with the fringe maxima positions as depicted by red lines. SEM, scanning electron microscopy. 
due to this thin heating zone underpins the physics for blistering and ejection of film as reported in references ${ }^{7-8,24-27}$ over varying film thickness without evidence of internal structuring of the transparent film. However, such interface machining was found together with the first evidence of internal structuring of the film as shown in Figure 1c by the SEM images for the present $500 \mathrm{~nm}$ thick film exposed at a threshold incident intensity of $I_{\text {avg }}=9 \times 10^{12} \mathrm{~W} \mathrm{~cm}^{-2}$. Laser-induced cleavage planes are seen to have ejected thin disk segments or formed nanovoids at positions found to align with the calculated positions of the interference maxima as indicated by the red connecting lines to Figure 1b. A radius of $0.53 \mu \mathrm{m}$ is observed for the fully ejected second disk in Figure $1 \mathrm{c}$, which is commensurate with the radius $\omega_{\mathrm{o}}=0.494 \mu \mathrm{m}\left(1 / e^{2}\right)$ calculated for the focused Gaussian beam. At this radial position $\left(\omega_{\mathrm{o}}\right)$, the internal laser intensity modulates axially from 0.84 to $3.74 \mathrm{TW} \mathrm{cm}^{-2}$ as shown in Figure $1 \mathrm{~b}$, suggesting a threshold intensity exposure of $3.74 \mathrm{TW} \mathrm{cm}^{-2}$ for internal structuring of the film.

The intensity profile (Figure 1b) was directly applied to predict the electron density profile generated inside the film at the observed threshold for internal structuring. Because of the short duration laser pulse, nonlinear light interactions inside the dielectric film will be dominated by multiphoton absorption and electron avalanche that ionize atoms to create an electron density $n_{e}$ according to Equation (1): ${ }^{28}$

$$
\frac{\mathrm{d} n_{e}(t)}{\mathrm{d} t}=n_{e}(t) w_{\mathrm{imp}}+N_{a} w_{\mathrm{mpi}}-\frac{n_{e}(t)}{\tau_{r}}
$$

Here, the impact ionization rate ( $\left.w_{\text {imp }}\right)$ and multiphoton ionization (MPI) rate $\left(w_{\mathrm{mpi}}\right)$ at the incident laser intensity $(I)$, are given, by Equations (2) and (3), respectively ${ }^{28}$

$$
\begin{gathered}
w_{\mathrm{imp}} \approx \frac{\varepsilon_{\mathrm{osc}}}{J_{i}} \frac{2 \omega^{2} v_{\mathrm{eff}}}{v_{\mathrm{eff}}^{2}+\omega^{2}} \\
w_{\mathrm{mpi}} \approx \omega N_{a}^{3 / 2}\left(\frac{\varepsilon_{\mathrm{osc}}}{J_{i}}\right)^{N}
\end{gathered}
$$

and the effective electron collision time $\left(\tau_{\text {eff }}\right)$ and the electron quiver energy $\left(\varepsilon_{\text {osc }}\right)$ are calculated by Equations $(4)^{29}$ and $(5):{ }^{28}$

$$
\begin{gathered}
\tau_{\text {eff }}[\mathrm{s}]=\frac{1}{v_{\text {eff }}}=\frac{16 \varepsilon_{o}^{2} \sqrt{m_{e}^{*}\left(0.1 E_{g}\right)^{3}}}{\sqrt{2} e^{4} n_{e}(t)} \\
\varepsilon_{\mathrm{osc}}[\mathrm{eV}]=\frac{e^{2} \varepsilon^{2}}{4 m_{e} \omega^{2}}=9.34 \frac{I}{10^{14}\left[\mathrm{~W} \mathrm{cm^{-2 } ]}\right.} \lambda^{2}[\mu \mathrm{m}]
\end{gathered}
$$

The electron relaxation $\left(\tau_{r}\right.$ term in Equation (1)) of $\sim 170 \mathrm{fs}^{30}$ is comparable with the short duration $\left(\tau_{\text {laser }}=200 \mathrm{fs}\right.$ ) laser pulse. However, electron avalanche in only the last $60 \mathrm{fs}$ of laser interaction was calculated to generate $90 \%$ of the ionization and electron relaxation was therefore insignificant in the calculation. For $\operatorname{SiN}_{\infty}$ values of $N_{a}=8 \times 10^{22} \mathrm{~cm}^{-3}$ for the atomic density, $E_{g}=5.3 \mathrm{eV}$ for the bandgap, ${ }^{31} J_{i}=E_{g}$ for the ionization potential and $m_{e}{ }^{*}=m_{e}$ for the effective mass of electron were used for computing the electron density. The laser frequency is given by $\omega=2 \pi c / \lambda$ and the order of nonlinear MPI, $N=\left\lceil\mathrm{J}_{\mathrm{i}} / \hbar \omega\right\rceil=3$.

The time-dependent Equations (1), (2) and (4) were simultaneously solved to follow the temporal rise of the electron density expected for the spatial intensity profile in Figure 1b. At the end of the laser pulse, the electron density is seen in Figure $1 \mathrm{~b}$ to peak strongly at the fringe maxima to a value of $5.87 \times 10^{21} \mathrm{~cm}^{-3}$. This value surpasses the critical plasma density $\left(n_{\mathrm{cr}} \sim 4.10 \times 10^{21} \mathrm{~cm}^{-3}\right)$ where the plasma becomes opaque to the laser and is typically expected to initiate material damage. ${ }^{23,28,29}$ Hence, the radial extent of the ejected disks at the laser-defined cleavage planes $(0.494 \mu \mathrm{m}$ in Figure $1 \mathrm{c})$ match closely with the typical laser-plasma conditions found to damage materials. Further, the simulations showed that impact ionization narrows the laser-plasma zone to $45 \mathrm{~nm}$ thick disks in Figure $1 \mathrm{~b}$ that is significantly narrower than the Fabry-Perot fringe width of $91 \mathrm{~nm}$. Further consideration of rapid thermal diffusion from such localized heating zones on such short fringe-to-fringe spacing $\left(\lambda / 2 n_{f}\right)$ is anticipated in very short time scales, $\tau_{d}=\lambda^{2} / 64 n_{f}^{2} D$ that fall in the picosecond to nanosecond range, as found by equating the thermal diffusion scale length, $\sqrt{4 D \tau_{d}}$, in a film having thermal diffusivity, $D$, with one-half of the fringe spacing $\left(\lambda / 4 n_{f}\right)$. Hence, for the present 200 fs laser interaction, an array of thin heating disks are predicted to have formed (Figure 1a) on the $\lambda / 2 n_{\text {film }}$ fringe spacing on time scales shorter than thermal transport and thereby, serve as a new means for machining inside transparent film on size scales much smaller than the $\sim 2.3 \mu \mathrm{m}$ depth of focus.

\section{RESULTS AND DISCUSSION}

The definitive evidence of the confinement of the laser-generated plasma into thin disks to create sharp and periodic cleavage planes inside the film is the observed alignment of the annular structures, the ejected membranes and the nanovoids in Figure 1c with the calculated fringe maxima positions in Figure 1b. The ejection of the first membrane structure (segment 1 , see Figure $1 b, S 1$ ) is evidenced by the annular ring seen in the top view at $\sim 34 \mathrm{~nm}$ depth that matches closely with the expected $29 \mathrm{~nm}$ deep position of the first fringe. The partially attached membrane (segment 2, see Figure 1b, S2) was formed by plasma-cleavage at the first and second fringe maxima, defining a $135( \pm 5) \mathrm{nm}$ thick membrane that matches the expected $\lambda / 2 n_{\text {film }}=131.8 \mathrm{~nm}$ fringe spacing. The third and fourth expected membrane structures (segments 3 and 4, see Figure 1b, S3 and S4) are seen to be fused into a double layer of 267 $( \pm 5) \mathrm{nm}$ thickness to form a non-punctured blister with thickness that matches the expected double-fringe spacing $\left(2 \lambda / 2 n_{\text {film }}=263.8 \mathrm{~nm}\right)$. Underlying this blister, a microexplosion from a thin disk plasma zone is inferred to have expanded into an $\sim 800 \mathrm{~nm}$ diameter nanovoid of $138( \pm 5)$ nm height. A deeper nanovoid is seen to have opened at the silicon/film interface to $45( \pm 5) \mathrm{nm}$ height. These nanovoids define the fifth and final membrane (segment 5, see Figure 1b, S5) whose observed $64( \pm 5) \mathrm{nm}$ thickness matches closely with the expected quarter-fringe thickness $(65.7 \mathrm{~nm})$. One may therefore understand the fusion of the third and fourth segments to be an anomaly associated with opposing forces of microexplosions in the top two cleavage planes against the powerful shock and pressure driven from laser microexplosions at the fourth fringe position and the film/silicon interface.

Once critical plasma density is reached at the first fringe position, strong light reflection and attenuation will reduce the forward propagating beam intensity, diminishing the interaction strength at deeper fringe positions. This presents the opportunity for controlling the number of laser-heating zones to vary the number of ejected segments and nanovoids formed inside the film with varying laser exposure. These principles were examined for a thicker $945 \mathrm{~nm} \mathrm{SiN}$ film in Figure 2 for the Gaussian-shaped beam to the maximum available fluence of $21.65 \mathrm{~J} \mathrm{~cm}^{-2}$. The top and cross-sectional SEMs (Figure 2a(i)-(viii)) show an expected widening of the laser modification zone for such beam shape from $1.3 \mu \mathrm{m}$ to $2 \mu \mathrm{m}$ diameter with the increasing fluence. Near the modification threshold of $3.50 \mathrm{~J} \mathrm{~cm}^{-2}$, a first segment of $64( \pm 5) \mathrm{nm}$ thickness was completed ejected while segment 2 (see Figure 1b, S2) formed into a punctured blister with a 


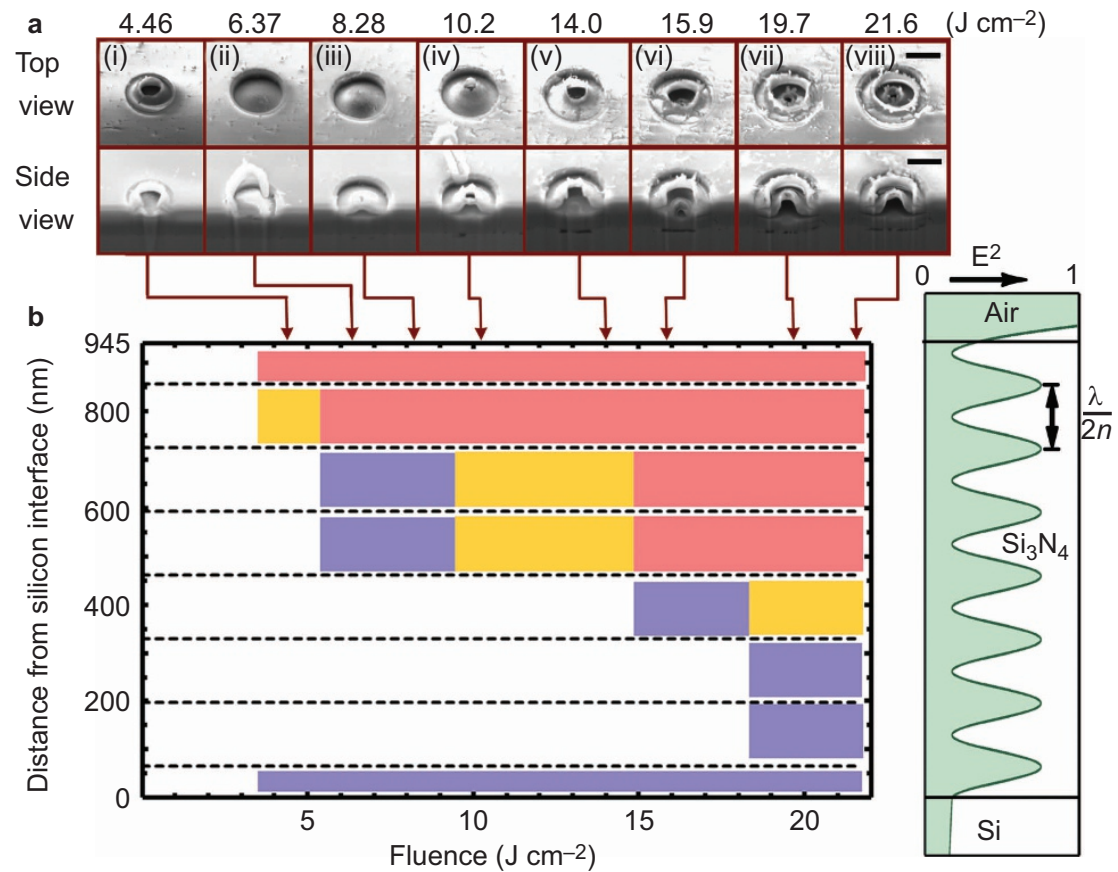

Figure 2 The quantized ejection of multiple segments in a $945 \mathrm{~nm} \mathrm{SiN}$ film as observed with increasing fluence for a Gaussian-shaped beam to a maximum available fluence of $21.65 \mathrm{~J} \mathrm{~cm}^{-2}$, as shown in top and cross-sectional SEMs (a(i)-(viii)). Slightly above threshold at $4.46 \mathrm{~J} \mathrm{~cm}{ }^{-2}$ fluence (a(i)), the first and second segments are ejected and blistered, respectively, and damage is evident at the $\mathrm{SiN}_{x} /$ silicon interface. The sequence of blistering, puncturing and ejection of each segment with increase in laser fluence is summarized graphically in (b) by the threshold fluences observed to form a solid blister (purple), a punctured blister (yellow) and an ejected blister (orange-red). Each segment was found to align between the cleavage planes (horizontal dashed lines) predicted by the positions of the Fabry-Perot intensity maxima shown graphically on the right. SEM, scanning electron microscopy.

$\sim 135 \mathrm{~nm}$ diameter open hole. At a higher fluence of $6.37 \mathrm{~J} \mathrm{~cm}^{-2}$, Figure $2 \mathrm{a}$ (ii) shows the complete ejection of segment 2 (see Figure $1 \mathrm{~b}$, S2). The third and fourth segments are seen (side view in Figure $2 \mathrm{a}$ (iii)) here to be fused into a $270( \pm 5) \mathrm{nm}$ thick blister overlying a $260( \pm 5)$ $\mathrm{nm}$ deep nanovoid, as similarly observed in the previous case of the $500 \mathrm{~nm}$ film. These fused segments form into a punctured blister at $10.2 \mathrm{~J} \mathrm{~cm}^{-2}$ fluence (side view in Figure $2 \mathrm{a}(\mathrm{iv})$ ) and are partially ejected at the higher laser exposure of $15.9 \mathrm{~J} \mathrm{~cm}^{-2}$ (side view in Figure $2 \mathrm{a}(\mathrm{vi})$ ), leaving an annular ledge clearly visible within the via shown in Figure $2 \mathrm{a}$ (vii). This sequence of blistering, puncturing and ejection of segments to quantized depths advances deeper inside the film with further increase in laser fluence (i.e., Figure $2 \mathrm{a}(\mathrm{vi})-($ viii))

The developing morphology with increasing fluence is summarized graphically in Figure $2 \mathrm{~b}$ by the threshold fluences observed to form a solid blister (purple), a punctured blister (yellow) and an ejected blister (orange-red) and were aligned vertically for each segment according to the observed cleavage plane (dashed line). The cleavage positions were again found to align closely $(\leqslant \pm 6 \mathrm{~nm})$ with the calculated Fabry-Perot intensity maxima as aligned graphically on the right. Segment 1 (see Figure 1b, S1) was found to eject at $4.46 \mathrm{~J} \mathrm{~cm}^{-2}$ threshold fluence (orange-red), together with the perforated blistering of segment 2 (see Figure 1b, S2) (yellow) without revealing a blistering phase for the first segment. The laser-plasma generated at the first fringe may have burnt through the thin first segment $(64 \mathrm{~nm})$ to prevent such blistering. A nanovoid was not observed to open between segments 3 and 4 (see Figure 1b, S3 and S4), resulting in blistering, perforation and ejection of these fused segments in the respective fluence ranges of 5.41-9.24 $\mathrm{J} \mathrm{cm}^{-2}, 9.24-14.97 \mathrm{~J} \mathrm{~cm}^{-2}$ and $\geqslant 14.97 \mathrm{~J} \mathrm{~cm}^{-2}$, respectively, as shown in Figure $2 \mathrm{a}$ (ii)-(vi) and $2 \mathrm{~b}$. This anomalous fusion was observed also in films varying from $500 \mathrm{~nm}$ a ICCD delay time (ns)

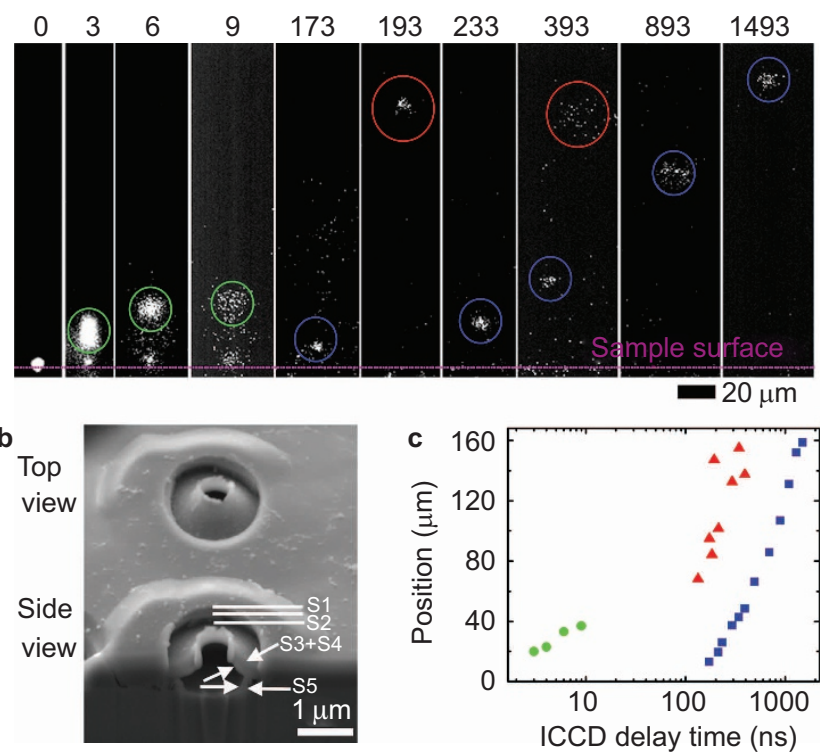

Figure 3 Time-resolved ICCD images (a) of ablation plumes recorded transversely from a $500 \mathrm{~nm} \mathrm{SiN}$ film exposed to $13.67 \mathrm{~J} \mathrm{~cm}^{-2}$ fluence and the top and cross-sectional SEMs (b) of the film after exposure. The plume emissions (a) appear in several clusters associated with the ejection of segments 1 (S1) and 2 (S2) (green) in the 3-9 ns window, partial ejection of fused segments 3 (S3) and 4 (S4) (red) in 133-393 ns window and ejection of segment 5 (S5) (blue) in 1731493 ns window. The observed position of clusters (c) from the surface as a function of time. ICCD, intensified CCD camera; SEM, scanning electron microscopy; S1-S5: see Figure 1b, S1-S5. 
to $1545 \mathrm{~nm}$ thickness. Figure $2 \mathrm{a}(\mathrm{i})$ and $2 \mathrm{~b}$ also shows the early onset of void formation at the $\mathrm{SiN}_{x} / \mathrm{Si}$ interface at a low fluence threshold of $3.50 \mathrm{~J} \mathrm{~cm}^{-2}$.

The observed remains of the ejected $\mathrm{SiN}_{x}$ segments (Figures $1 \mathrm{c}$, $2 \mathrm{a}$ (ii) and $2 \mathrm{a}(\mathrm{vi})$ ) suggest that the array of laser-induced plasma zones do not burn through and vaporize the forming membranes. Thus, one anticipates the quantized ejections of segments in a temporal sequence as the plasma planes heat and microexplode, beginning from the nearsurface to the lower cleavage positions as depicted in Supplementary Movie S1. The evidence for this sequential ejection is seen in time-gated ICCD images recorded from a $500 \mathrm{~nm} \mathrm{SiN}_{x}$ film shown in Figure 3, revealing time-delayed repeating ejections of ablation plume whose number matched well with the number of segments found by SEM to be ejected for a given laser fluence. For example, the SEM image of the film (Figure 3b) reveals the ejection of five segments when irradiated with $13.67 \mathrm{~J} \mathrm{~cm}^{-2}$ fluence. The clusters of plume were observed appearing in distinct time zones of 3-9 ns (green), 133-393 ns (red) and 1731493 ns (blue), marked in Figure 3a. The plume positions were followed up to $160 \mu \mathrm{m}$ distance from the film surface, with their observed positions recorded as a function of time in Figure $3 c$. We infer the segment 5 (see Figure 1b, S5) to be the last ejected plume (blue), which was fully ejected according to Figure $3 \mathrm{~b}$. This figure also indicates only a fractional ejection of the fused third and fourth segments (see Figure 1b, S3 and S4) (red), which appear indistinguishable and faint in ICCD image frames at 133-393 ns in Figure 3a. The fused segments appear together with the segment 5 (see Figure 1b, S5) at $\sim 393$ ns, but being ejected forward more quickly than the segment 5 (see Figure 1b, S5). Therefore, the bright emissions observed in the 3-10 ns zone are ascribed to plume expansion and membrane ejection of the segments 1 and 2 (see Figure 1b, S1 and S2) promptly after the laser exposure. At this fluence, the first two segments (see Figure 1b, S1 and S2) appeared bright and promptly, moving at $\sim 2.9 \mathrm{~km} \mathrm{~s}^{-1}$ speed, while the ejection of deeper layers were seen much later (173-1493 ns) owing to much lower speeds, for example, of $0.1 \mathrm{~km} \mathrm{~s}^{-1}$ for the segment 5 (see Figure $1 \mathrm{~b}, \mathrm{~S} 5$ ) as inferred from Figure $3 \mathrm{c}$. Hence, the directly ablated surface material and first ejected segments appear promptly with the highest speeds, while the inertia of pushing against the upper layers leads to much delayed ejection and $\sim 30$-fold lower ejection speeds for the deeper segments of the film.

\section{CONCEPTS FOR THIN FILM DEVICES}

The combination of quantized surface ejection and nanovoid formation directly inside a thin transparent film opens a new means for fabricating novel combinations of optical, nanofludic and MEMS components with facile delivery of varying laser exposure. Figure $4 \mathrm{a}$

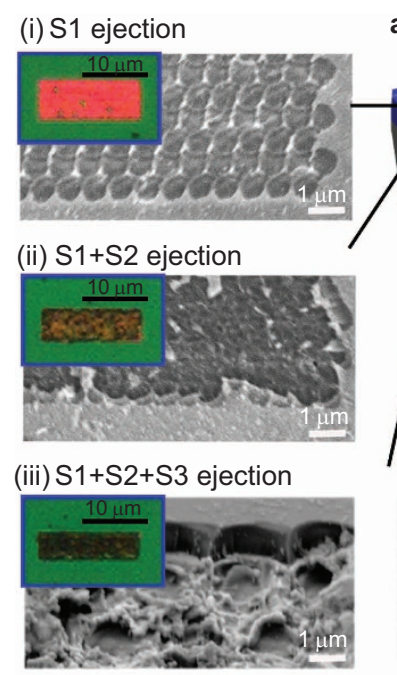

a

$$
\text { (iv) Multi-level ejections }
$$

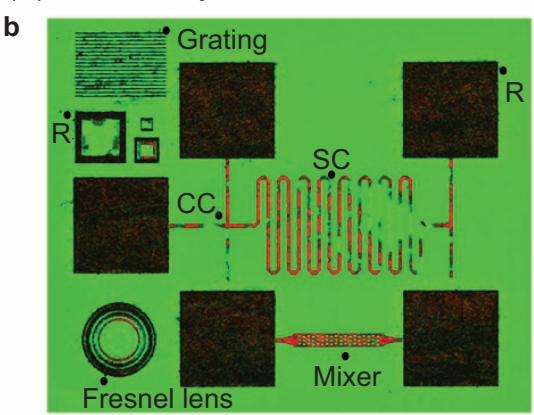

(vii) Fresnel lens

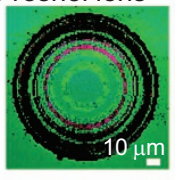

(vi) Blazed grating

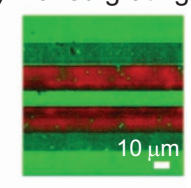

(v) Mixer with

embedded barriers

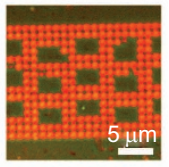

viii) Buried channel

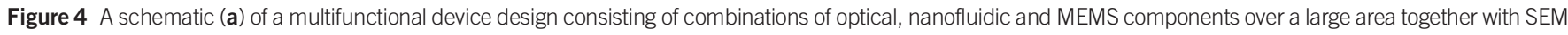

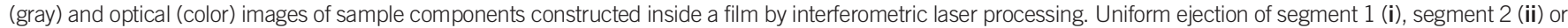

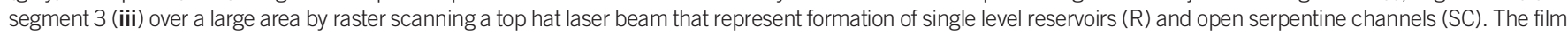

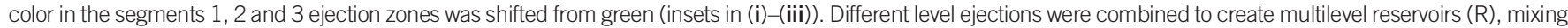

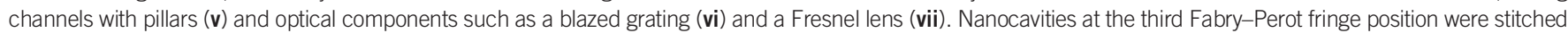

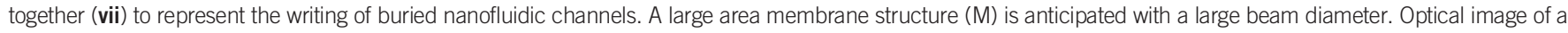

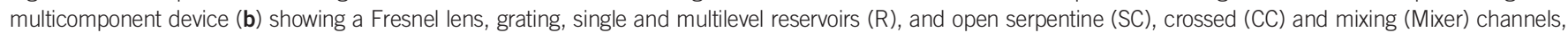
fabricated with interferometric laser fabrication. MEMS, micro-electro-mechanical system; SEM, scanning electron microscopy; S1-S3: see Figure 1b, S1-S3. 
presents this concept for fabricating micro- and nanofluidic devices that include various reservoir designs connected with different types of open and buried channels, including a mixing channel with embedded barriers and serpentine channels. Fresnel lenses and blazed gratings are further depicted together with a large area membrane sensor. The laser-structured devices may potentially be fabricated in films coated over microelectronic and CCD devices on silicon wafers that collectively offer a flexible and attractive integration platform.

Expanding the interferometric fabrication principle to larger processing area may be approached by scaling up the pulse energy and blistering the film into a large-diameter MEMS device (M) as identified in Figure $4 \mathrm{a}$. However, such uniform processing requires focusing to a flattop beam profile. With the present laser system, a uniform beam was only available up to a maximum radius of $\sim 0.75 \mu \mathrm{m}$, which yielded more uniform ejected structure as presented in Supplementary Fig. S2. Structuring beyond this focal $1.5 \mu \mathrm{m}$ spot size was therefore approached by stitching together arrays of individual exposure spots formed with the uniform square beam. Various grid patterns were examined at variable laser exposures to optimize this stitching and generate a uniform morphology over a larger area as shown in Supplementary Fig. S3. In this way, individual laser ejection zones to the first, second or third segments could be stitched with high reproducibility over large scanned areas to depths aligned closely with the expected fringe positions at $29 \mathrm{~nm}, 161 \mathrm{~nm}$ and $293 \mathrm{~nm}$ depths, respectively, as shown in Figure $4 \mathrm{a}(\mathrm{i})-(\mathrm{iii})$, respectively. Interestingly, ejection of only the first segment was observed here when patterned in closely packed arrays and exposed below the ejection threshold for an isolated laser spot. Laser shock may be dislodging weakly bonded first segments in the neighboring exposure sites. The connected array of these first segment ejected spots offered the smoothest morphology, manifesting in the expected color shift by thin-film interference from green of the deposited $500 \mathrm{~nm}$ film to red for the remaining $471 \mathrm{~nm}$ film as seen by the inset image of Figure 4a(i) and characterized in Supplementary Fig. S4. However, an increasing amount of ablation debris was observed with deeper ejected segments that is most prominent in Figure $4 \mathrm{a}$ (iii) for the third segment ejection. This nanoscale debris leads to strong optical scattering, overshadowing the thin-film interference effect to give the grey coloring seen in the case of the second and third segment ejections (Figure $4 \mathrm{a}$ (ii) and $4 \mathrm{a}$ (iii) insets, respectively).

Regardless of this roughness, different ejection levels could be interfaced to form pillars as shown in Figure $4 a$ (iv). This multilevel structuring was applied to demonstrate the concept of a microfluidic mixer with embedded barriers, a Fresnel optical lens and a blazed optical grating as shown in the optical images of Figure $4 \mathrm{a}(\mathrm{v})-(\mathrm{vii})$, respectively. The integration of these fabrication principles is demonstrated in Figure $4 b$, where deep reservoirs (R) were connected with various crossed (CC), serpentine (SC) and mixing open channels (Mixer), written in a single run of three laser exposures to pattern over three different ejection levels. This microscope image clearly reveals the anticipated color changes with the level of ejected segment.

As previously seen (Figures 1 and 2), nanovoids are expected to have opened inside the film below the ejected segment layers (Figure 4a(i)-(iii)). The potential for linking the buried nanovoids below a closed-packed array of exposure spots is clearly demonstrated at the third fringe position in Figure $4 \mathrm{a}$ (viii), thus opening the means for writing buried nanofluidic channels that may link the various reservoirs as proposed in Figure 4a.

\section{CONCLUSIONS}

We report a novel method for highly resolved axial processing inside a thin dielectric film on a high index substrate with a femtosecond laser by confining laser-material interaction to an array of narrow zones inside the film as was shown in Figure 1a. This confinement is anticipated in transparent films of thickness $\geqslant \lambda / 4 n_{\text {film }}$, where the optical interference of Fresnel reflections from air/film and film/substrate interface creates a Fabry-Perot intensity modulation of the laser light on $\lambda / 2 n_{\text {film }}$ fringe spacing (Figure $1 \mathrm{~b}$ ). Nonlinear laser interactions predicted the electron density profile to narrow to $45 \mathrm{~nm}$ thick plasma disks that are more than 50-fold narrower than the laser depth of focus. At the threshold exposure for internal structuring, the electron density reaches critical at the predicted fringe maxima positions to facilitate the quantized ejection of the film or the formation of thin nanovoids inside the film at these laser cleaving planes as shown in Figures 1 and 2. This geometry for internal laser cleaving has not been previously reported inside a transparent material and greatly extends the control over the laser modification in contrast with internal structuring over the whole laser focal volume ${ }^{6}$ or structuring confined at a film/substrate interface. ${ }^{9,25-27,32}$ Further, the predicted plasma disks were shown by intensified CCD imaging (Figure 3 ) to validate the quantized ejection of multiple segments in a temporal sequence. Both internal structuring and quantized ejection of films was observed in 500-1500 nm thick films with either uniform (Supplementary Fig. S2) or Gaussian beam (Figures 1-3) shape.

This partial and digital removal of a thin transparent film opens new directions in selectively texturing and surface micromachining to $\lambda /$ $2 n_{\text {film }}$ precision inside the film and in finely pitched patterns of $1 \mu \mathrm{m}$ lateral resolution. This opens new means for marking, coloring and multilevel structuring of thin transparent films (Figure 4 and Supplementary Fig. S3), as well as finer control in the material transfer underlying laser-induced forward transfer, ${ }^{10}$ matrix-assisted pulsed laser evaporation-direct write $^{14}$ and catapulting ${ }^{13}$ applications. Further, the combination of this laser-direct writing of multilevel patterns with buried nanovoids or channels can be exploited as demonstrated in Figure 4 for fabricating MEMS, optofluidic and other optical components in thin films on wafers or flexible silicon foils. Lastly, the formation of thin membrane blisters with or without perforations offers a new platform for biological studies and creation of mesoscopic materials. This combination of blistering and ejection is very attractive for transforming lab-on-a-chip devices to flexible labin-a-film structures that are compatible with today's state-of-the-art manufacturing facilities for CMOS microelectronics or glass display and create novel chip-scale biosensors, minimally invasive implantable devices, portable point-of-care medical products, compact diagnostic platforms or interactive sensor display. One can envision such laser-structured films over microelectronic chips, light sources or optical sensors, and ultrathin wafers to create epidermal biosensors in ultrathin foldable and stretchable integrated circuits or to shape vascular-type networks into bio-implants that mimics natural structures.

\section{ACKNOWLEDGMENTS}

We thank A Khalatpour for assistance with the thin film optical interference modeling. This work was supported by the Natural Sciences and Engineering Research Council of Canada, the Ontario Research Fund-Research Excellence program and the University of Toronto.

1 Küper S, Stuke M. Femtosecond UV excimer laser ablation. App/ Phys B 1987; 44 199-204.

2 Mourou G, Du D, Dutta SK, Elner V, Kurtz R et al. Method for controlling configuration of laser induced breakdown and ablation. US Patent RE37585 E. 2002 Mar 19. 
3 Schermelleh L, Thalhammer S, Heckl W, Pösl H, Cremer T et al. Laser microdissection and laser pressure catapulting for the generation of chromosome-specific pain probes. Biotechniques 1999; 27: 362-367.

4 Miura K, Qiu J, Inouye H, Mitsuyu T, Hirao K. Photowritten optical waveguides in various glasses with ultrashort pulse laser. Appl Phys Lett 1997; 71: 3329-3331.

5 Gattass RR, Mazur E. Femtosecond laser micromachining in transparent materials. Nat Photon 2008; 2: 219-225.

6 Glezer EN, Mazur E. Ultrafast-laser driven micro-explosions in transparent materials. Appl Phys Lett 1997; 71: 882-884.

7 McDonald JP, Thouless MD, Yalisove SM. Mechanics analysis of femtosecond laserinduced blisters produced in thermally grown oxide on Si(100). J Mater Res 2010; 25: 1087-1095.

8 Rublack T, Hartnauer S, Kappe P, Swiatkowski C, Seifert G. Selective ablation of thin $\mathrm{SiO}_{2}$ layers on silicon substrates by femto- and picosecond laser pulses. Appl Phys $A$ 2011; 103: 43-50.

9 McDonald JP, Mistry VR, Ray KE, Yalisove SM. Femtosecond pulsed laser direct write production of nano- and microfluidic channels. Appl Phys Lett 2006; 88: 183113.

10 Pique A. Rapid Prototyping of Embedded Microelectronics. In: Hoque ME, editor. Rapid Prototyping Technology_Principles and Functional Requirements. Rijeka: InTech; 2011. pp247-272.

11 Kumar K, Lee KK, Herman PR, Nogami J, Kherani NP. Femtosecond laser direct hard mask writing for selective facile micron-scale inverted-pyramid patterning of silicon. Appl Phys Lett 2012; 101: 222106.

12 Bohandy J, Kim BF, Adrian FJ. Metal deposition from a supported metal film using an excimer laser. J App/ Phys 1986; 60: 1538-1539.

13 Tolbert WA, Lee IS, Doxtader MM, Ellis EW, Dlott DD. High-speed color imaging by laser ablation transfer with a dynamic release layer: fundamental mechanisms. J Image Sci Technol 1993; 37: 411-421.

14 Piqué, A. Chrisey DB, Auyeung RC, Fitz-Gerald J, Wu HD et al. A novel laser transfer process for direct writing of electronic and sensor materials. App/ Phys A 1999; 69 S279-S284.

15 Westphal G, Burgemeister R, Friedemann G, Wellmann A, Wernert N et al. Noncontact laser catapulting: a basic procedure for functional genomics and proteomics. Meth Enzymol 2002; 356: 80-99.

16 Hill KO, Fujii Y, Johnson DC, Kawasaki BS. Photosensitivity in optical fiber waveguides: application to reflection filter fabrication. Appl Phys Lett 1978; 75 : 647-649.

17 Campbell M, Sharp DN, Harrison MT, Denning RG, Turberfield AJ. Fabrication of photonic crystals for the visible spectrum by holographic lithography. Nature 2000, 404: 53-56.

18 Kondo T, Yamasaki K, Juodkazis S, Matsuo S, Mizeikis V et al. Three-dimensional microfabrication by femtosecond pulses in dielectrics. Thin Solid Films 2004; 453454: 550-556.
19 Hosokawa Y, Yashiro M, Asahi T, Masuhara H, Kadota T et al. Femtosecond multistep laser etching of transparent amorphous organic film. Jpn J Appl Phys 2001; 40: L1116-L1118.

20 Hosokawa Y, Yashiro M, Asahi T, Masuhara H. Photothermal conversion dynamics in femtosecond and picosecond discrete laser etching of $\mathrm{Cu}$-phthalocyanine amorphous film analysed by ultrafast UV-VIS absorption spectroscopy. J Photochem Photobiol A 2001; 142: 197-207.

21 Mero M, Sabbah AJ, Zeller J, Rudolph W. Femtosecond dynamics of dielectric films in the pre-ablation regime. Appl Phys A 2005; 81: 317-324.

22 Mero M, Clapp B, Jasapara JC, Rudolph W, Ristau D et al. On the damage behavior of dielectric films when illuminated with multiple femtosecond laser pulses. Opt Eng 2005; 44: 051107.

23 Jasapara J, Nampoothiri AV, Rudolph W. Femtosecond laser pulse induced breakdown in dielectric thin films. Phys Rev B 2001; 63: 045117.

24 Rublack T, Schade M, Muchow M, Leipner HS, Seifert G. Proof of damage-free selective removal of thin dielectric coatings on silicon wafers by irradiation with femtosecond laser pulses. J Appl Phys 2012; 112: 023521.

25 Tull BR, Carey JE, Mazur E, McDonald JP, Yalisove SM. Silicon surface morphologies after femtosecond laser irradiation. MRS Bull 2006; 31: 626-633.

26 McDonald JP, McClelland AA, Picard YN, Yalisove SM. Role of a native oxide on femtosecond laser interaction with silicon (100) near the damage threshold. App Phys Lett 2005; 86: 264103.

27 McDonald JP, Mistry VR, Ray KE, Yalisove SM, Nees JA et al. Femtosecond-laserinduced delamination and blister formation in thermal oxide films on silicon (100). Appl Phys Lett 2006; 88: 153121.

28 Gamaly EG, Rode AV, Luther-Davies B, Tikhonchuk VT. Ablation of solids by femtosecond lasers: ablation mechanism and ablation thresholds for metals and dielectrics. Phys Plasmas 2002; 9: 949-957.

29 Jing XF, Tian Y, Zhang JC, Chen SL, Jin YX et al. Modeling validity of femtosecond laser breakdown in wide bandgap dielectrics. App/ Surf Sci 2012; 258: 4741-4749.

30 Sun $\mathrm{Q}$, Jiang HB, Liu Y, Wu ZX, Yang H et al. Measurement of the collision time of dense electronic plasma induced by a femtosecond laser in fused silica. Opt Lett 2005; 30: 320-322.

31 Wang Y, Shen DZ, Liu YC, Zhang JY, Zhang ZZ et al. Visible photoluminescence of Si clusters embedded in silicon nitride films by plasma-enhanced chemical vapor deposition. Physics E 2005; 27: 284-289.

32 Rublack T, Seifert G. Femtosecond laser delamination of thin transparent layers from semiconducting substrates. Opt Mater Express 2011; 1: 543-550.

\footnotetext{
(c) (1) (2) This work is licensed under a Creative Commons Attribution-

BY NA NA NonCommercial-ShareAlike 3.0 Unported license. To view a copy of this license, visit http://creativecommons.org/licenses/by-nc-sa/3.0
} 